\title{
Heterogeneous integration in silicon photonics through micro-transfer-printing
}

\author{
Jing Zhang( ${ }^{(1)}$, Grigorij Muliuk ${ }^{(1)}$, Jeroen Goyvaerts ${ }^{(1)}$, Bahawal Haq ${ }^{(1)}$, Alexandros Liles ${ }^{(1)}$, Sulakshna Kumari ${ }^{(1)}$, Joan Juvert( ${ }^{(1)}$, \\ Camiel Op de Beeck ${ }^{(1)}$, Bart Kuyken ${ }^{(1)}$, Joris Van Campenhout ${ }^{(2)}$, Guy Lepage ${ }^{(2)}$, Peter Verheyen ${ }^{(2)}$, Agnieszka Gocalinkska ${ }^{(3)}$, \\ Emanuele Pelucchi ${ }^{(3)}$, Brian Corbett ${ }^{(3)}$, Antonio Jose Trindade ${ }^{(4)}$, Chris Bower ${ }^{(4)}$, and Gunther Roelkens ${ }^{(1)}$ \\ (1) Ghent University - imec \\ (2) IMEC \\ (3) Tyndall National Institute \\ ${ }^{(4)} \mathrm{X}$-Celeprint limited \\ (1) Technologiepark-Zwijnaarde 126, 9052 Ghent, Belgium jingzhan.Zhang@UGent.be
}

\begin{abstract}
Micro-transfer-printing enables the intimate integration of a wide range of opto-electronic micro-components on a silicon photonics platform. This technique allows for wafer-scale integration in a massively parallel manner with high alignment accuracy, high throughput and high yield, therefore leading to a cost reduction of complex photonic integrated circuits.
\end{abstract}

\section{Introduction}

Silicon photonics $(\mathrm{SiPh})$ is emerging as the most promising contender for building ultra-compact integrated photonic circuits due to its high refractive index contrast, and has shown a spectacular development in the last two decades. The CMOS compatibility enables leveraging the well-established processes and mature fabrication tools of the CMOS industry, resulting in a high-volume and low-cost production of photonic integrated circuits (PICs) on $200 \mathrm{~mm}$ or $300 \mathrm{~mm}$ wafers. Over the past years, a variety of passive devices as well as $\mathrm{Si} / \mathrm{Ge}$-based active devices have been demonstrated on the $\mathrm{SiPh}$ platform, enabling complex and powerful PICs, for applications in optical interconnects, optical sensors, bio-medical instruments, etc. Nevertheless, additional integrated functionalities, especially in the form of optical sources, are desired for an improved performance while maintaining a compact footprint. Different approaches have been followed to realize the integration of non-inherent functionalities on Si PICs.

In case of the integration of III-V-on-Si devices/PICs, the monolithic integration through direct epitaxial growth of III-V semiconductors on the $\mathrm{Si}$ substrate, is considered to be the most ideal approach, as it allows for large scale manufacturing and thus low cost. Despite the substantial progress that has been made recently, this integration method is still at an early stage and hardly available for the demonstration of practical devices, especially when they need to be integrated with a silicon PIC. Further to the aforementioned approach, heterogeneous integration through wafer-to-wafer or dieto-wafer bonding has been widely adopted. This technique allows the integration of III-V material on a non-native substrate (e.g. silicon-on-insulator (SOI)) at wafer scale. Simultaneous integration of different III-V materials on the same substrate is possible, but still challenging. Moreover, it requires the modification of the back-end process flow, if $\mathrm{Si}$ and/or Ge based active devices are to be included in the PICs.

Micro-transfer-printing is a novel technology that has recently attracted considerable attention for the integration of micro-components in heterogeneous systems. This technique successfully tackles the above described issues in the wafer/die to wafer bonding technology, while maintaining the advantage of high throughput integration. Moreover, this approach allows for the pre-fabrication and pre-testing of the devices on their native substrate prior to their integration on a $\mathrm{Si}$ PIC, thus leading to a higher yield.

In this paper we discuss the demonstration of the heterogeneous integration of various opto-electronic components on $\mathrm{SiPh}$ platforms through the microtransfer-printing approach.

\section{Micro-transfer-printing process}

The concept of the micro-transfer-printing is schematically illustrated in Fig.1, taking the integration of III-V-on-silicon devices as an example. In microtransfer-printing a polydimethylsiloxane (PDMS) stamp is use to pick up (arrays of) pre-fabricated devices from a native substrate and print them on a $\mathrm{SiPh}$ target wafer. As depicted in Fig. 1(b), a sacrificial layer is required to enable the release of devices from its native substrate [1].

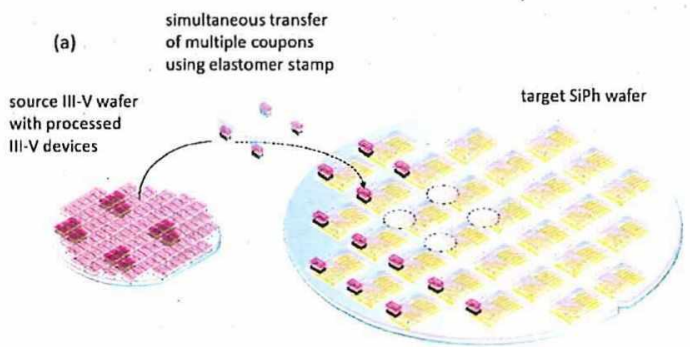

(b)

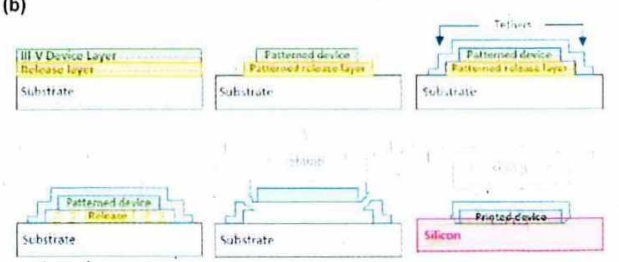

Fig. 1. (a) Schematic of transfer-printing-based integration on $200 \mathrm{~mm}$ or $300 \mathrm{~mm}$ silicon photonic wafer in a parallel manner; (b) prefabrication of III-V devices on its native substrate and transfer printing operation. 


\section{Micro-transfer printing of opto-electronic components onto PICs}

Fig. 2(a-c) shows some examples of the micro-transferprinting-based integration of different types of III-V photodetectors (PDs) on PICs. A yield up to $98.8 \%$ (83 of 84 printed C-band III-V PDs) in a parallel $(2 \times 2)$ integration process was obtained (Fig. 2(a)). Fig. 2(b) shows the integration of an array of O-band III-V PDs on a PIC with Si ring modulators and Fig. 2(c) depicts GaAs metal-semiconductor-metal (MSM) photodetectors on $\mathrm{SiN}$ waveguide circuits. Besides III-V semiconductors, any other material/device that can be released from its native substrate are transfer-printable. As an example, a Ge waveguide PD was released by underetching the $2 \mu \mathrm{m}$ thick buried silicon dioxide layer of the SOI wafer and printed on a Si PIC (Fig. 2(d)).

Fig. 2 (a) A zoom-in microscope image of transfer-printed III-V-on-Si PD array; (b) InP based p-i-n PDs on full platform PIC; (c) GaAs MSM PDs integrated on a SiN waveguide circuit; (d) transfer-printed Ge PDs on a $220 \mathrm{~nm}$ thick passive $\mathrm{Si}$ waveguide circuit.

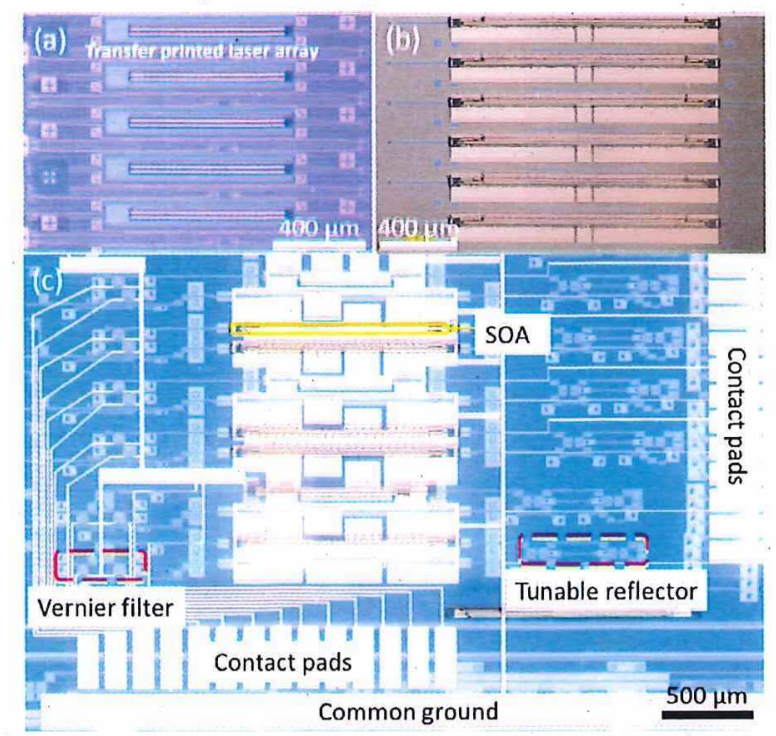

Fig. 3 Microscope image of an array of transfer printed etched facet Fabry-Perot lasers (a); (b) evanescently coupled SOAs; (c) widely tunable III-V-on-Si lasers.
As already mentioned, optical sources are urgently desired on Si PICs. Currently two major approaches are intensively studied for realizing integrated light sources through micro-transfer-printing. One route is by transfer printing a pre-fabricated III-V laser in a trench that reaches the silicon substrate. By using a co-designed spot size converter (e.g. a Si taper structure) in the PIC and thanks to a sub-micrometer alignment accuracy provided by the micro-transfer-printing system, milliwatt-level coupling to the silicon waveguide was successfully demonstrated (Fig. 3(a)). The other approach is based on evanescent coupling. As the commonly used III-V epitaxial materials have high refractive indices, $\mathrm{SiPh}$ platforms with a thicker silicon device layer (typically $400 \mathrm{~nm}$ ) are adopted, and a III-V adiabatic taper structure is used to enable the coupling between the III-V and the underlying Si layer. Fig. 3(b) shows an array of fabricated III-V-on-Si integrated SOAs, which have an up to $19 \mathrm{~dB}$ small signal gain at $190 \mathrm{~mA}$ bias current. By incorporating a pair of DBR reflectors at the sides of the SOAs, L-band single mode operation DBR lasers were realized with a maximum waveguide coupled output power over $6 \mathrm{~mW}$. Furthermore, III-V-on-Si integrated widely tunable lasers were also demonstrated in the same way. A microring-based Vernier filter is incorporated in the laser cavity to achieve a single mode operation and a wide tuning range. The threshold of the fabricated lasers is 70 $\mathrm{mA}$ and, the series resistance is found to be $15 \Omega$ at a bias current of $110 \mathrm{~mA}$. Over $48 \mathrm{~nm}$ tuning range is achieved by actuating both thermal heaters of the ring resonators, and the waveguide-coupled power varies between $-1.8 \mathrm{dBm}$ and $5.2 \mathrm{dBm}$ over the entire tuning range,

\section{Conclusion}

In this paper we summarized the heterogeneous integration of different types of opto-electronic components in PICs by means of micro-transfer-printing, which reveals the great potential that micro-transferprinting has for the realization of complex and advanced PICs.

\section{Acknowledgements}

This work was supported by the Methusalem funding of the Flemish government, by the $\mathrm{H} 2020$ project TOPHIT, TeraBoard, PIX4Life and CALADAN and the ECSEL project MicroPrince.

\section{References}

[1] B. Corbett, R. Loi, J. O'Callaghan, G. Roelkens, 'Transfer Printing for Silicon Photonics' in S. Lourdudoss, R. T. Chen, C. Jagadish,(Ed.): Semiconductors and Semimetals, Elsevier, 2018, pp 43-70. 


\section{THE TWENTY-FOURTH MICROOPTICS CONFERENCE}

\section{MOC2019}

\section{CONFERENCE PROGRAM}

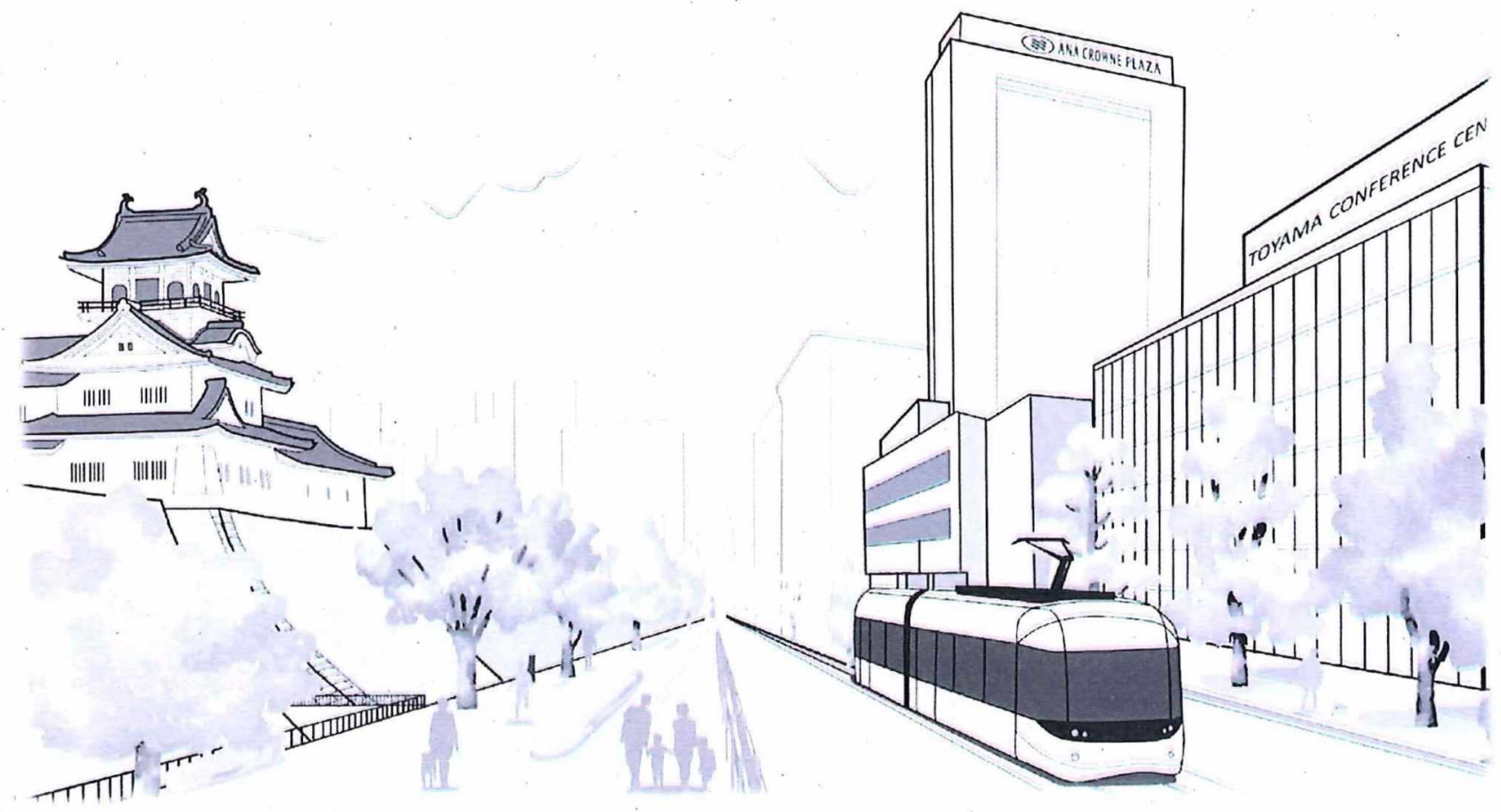

Sponsored by the Japan Society of Applied Physics (JSAP)

Organized by Microoptics Group, JSAP

Technically Cosponsored by IEEE Photonics Society

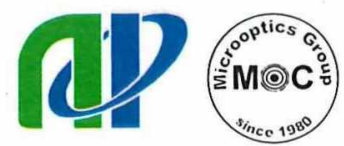

November 17 - 20, 2019

Toyama International Conference Center, Toyama, Japan 
9:00-10:45 Session C: Silicon Photonics

Chairs: S.-L. Huang, National Taiwan Univ.

R. Kou, A/ST

C-1 Cross-polarization effects in sheared two-dimensional grating couplers for silicon photonics

9:00 G. Georgieva', K. Voigt', C. Mai ${ }^{2}$, K. Petermann', and L. Zimmermann ${ }^{1.2},{ }^{1}$ TU Berlin, ${ }^{2} I H P$

C-2 Beam waist controlled vertical optical path conversion using integrated curved micro mirror for 9:15 silicon photonics

A. Noriki ${ }^{1,2}$ and T. Amano ${ }^{1,2},{ }^{1}$ AIST, ${ }^{2}$ PETRA

C-3 Reflective silicon arrayed waveguide grating using one-dimensional photonic crystal reflector

9:30 H. Okayama ${ }^{1,2}$, Y. Onawa ${ }^{1,2}$, H. Takahashi ${ }^{1,2}$, D. Shimura ${ }^{1,2}$, H. Yaegashi ${ }^{1,2}$, and H. Sasaki, ${ }^{1,},{ }^{1} R \& D$ Center, Oki Electric Industry Co. Ltd., ${ }^{2}$ Photonics Electronics Technology Research Association (PETRA)

C-4 Heterogeneous integration in silicon photonics through micro-transfer-printing (Invited)

9:45 J. Zhang', G. Muliuk', J. Goyvaerts', B. Haq', A. Liles', S. Kumari', J. Juvert', C. Op de Beeck', B. Kuyken',

J. Van Campenhout ${ }^{2}$, G. Lepage ${ }^{2}$, P. Verheyen ${ }^{2}$, A. Gocalinska ${ }^{3}$, E. Pelucchi ${ }^{3}$, B. Corbett ${ }^{3}$, A. J. Trindade ${ }^{4}$,

C. Bower', and G. Roelkens', 'Ghent University - imec, ${ }^{2}$ IMEC, ${ }^{3}$ Tyndall National Institute, ${ }^{4} X$-Celeprint limited

C-5 Novel Si photonic waveguides and applications to optical modulators (Invited)

10:15 S. Saito, M. Sotto, K. Debnath, J. Byers, A. Z. A.-Attili, I. Tomita, D. Burt, M. K. Husain, K. Ibukuro,

D. J. Thomson, W. Zhang, B. Chen, F. Y. Gardes, G. T. Reed, and H. N. Rutt, University of Southampton

Break (10:45-11:00)

11:00-12:45 Session D: Microoptics for Imaging (1)

Chairs: E. Acosta, Univ. of Santiago de Compostela

N. Mori, Yamashita Denso

D-1 Skin cancer detection using cellular resolution optical coherence tomography (Invited)

11:00 C.-K. Chang', J.-T. Lin', C. You', M. C.-Delgado', J.-W. Tjiu', and S.-L. Huang', 'National Taiwan University, ${ }^{2}$ National Taiwan University Hospital

D-2 Investigation of excitation beam modulation using azimuthal polarization to improve STED resolution 11:30 G. Lim', W.-C. Kim², and N.-C. Park', 'Yonsei University, ${ }^{2}$ Hanbat National University

D-3 Thin multi-aperture microscope

11:45 S. Schacke ${ }^{1,2}$, R. Berlich', B. Höfer', P. Dannberg', B. Zaage', E. Beckert ${ }^{1}$, and N. Danz ${ }^{1},{ }^{1}$ Fraunhofer Institute for Applied Optics and Precision Engineering IOF, ${ }^{2}$ Fraunhofer Project Hub for Microelectronic and Optical Systems for Biomedicine MEOS

D-4 Glass 3D printing for ultra-miniaturized endoscopic optical systems

12:00 S. Kretschmer, C. Ataman, and H. Zappe, University of Freiburg

D-5 Waveguide integrated organic laser source for lab-on-chip applications

12:15 M. Čehovski', J. Becker ${ }^{2}$, O. Charfi', R. Caspary', H.-H. Johannes', C. Müller', and W. Kowalsky', ${ }^{1} T U$ Braunschweig, IHF and Cluster of Excellence PhoenixD, ${ }^{2}$ University of Freiburg, FIT

D-6 Snapshot-type CCD spectrometer utilizing distributed passband-type multichannel photonic crystal

12:30 wavelength filter array

Y. Ohtera and N. Ikeda, Toyama Prefectural University

Lunch (12:45-13:45) 